\title{
Effect of initial stress on reflection at the free surface of anisotropic elastic medium
}

\author{
M D SHARMA \\ Department of Mathematics, Kurukshetra University, India 136119. \\ e-mail: mohan_here@rediffmail.com
}

\begin{abstract}
The propagation of plane waves is considered in a general anisotropic elastic medium in the presence of initial stress. The Christoffel equations are solved into a polynomial of degree six. The roots of this polynomial represent the vertical slowness values for the six quasi-waves resulting from the presence of a discontinuity in the medium. Three of these six values are identified with the three quasi-waves traveling in the medium but away from its boundary. Reflection at the free plane surface is studied for partition of energy among the three reflected waves. For post-critical incidence, the reflected waves are inhomogeneous (evanescent) waves. Numerical examples are considered to exhibit the effects of initial stress on the phase direction, attenuation and reflection coefficients of the reflected waves. The phase velocities and energy shares of the reflected waves change significantly with initial stress as well as anisotropic symmetry. The presence of initial stress, however, has a negligible effect on the phase directions of reflected waves.
\end{abstract}

\section{Introduction}

Crustal rocks are always subjected to stresses. The slow process of creep inside the Earth produces the initial stress which may be approximated as homogeneous near the surface (Biot 1940). Differential stress environment (McGarr 1980; Hanks and Raleigh 1980) in the crust is responsible for the preferential alignments in the Earth, ranging from mineral orientations, grains, or micro-cracks to regional fractures. The folds and fractures in the sedimentary layers are the result of differential stress environment in the sediments. The difference between confined tectonic stress and pore-fluid pressure conducts the flow of fluid between the connected cracks in a reservoir. This implies that the initial stress and crack-induced anisotropy not only coexist in the crust but will also be affecting the quality/quantity of each other.

In the presence of aligned cracks as well as initial stress, an elastic medium behaves anisotropic to wave propagation. The anisotropic behaviour of crustal rocks due to the presence of cracks and fractures may be explained through the anisotropic elastic constants (Crampin 1981). But the effect of initial stress on waves cannot be accounted by the elastic anisotropy or the change in elastic constants (Biot 1940). Fibre-reinforced composite materials are used in important structures due to their low weight and high strength. Such materials are a man-made example of pre-stressed elastic solids. In these materials, however, the effect of pre-stress is translated into their anisotropic behaviour to wave propagation (Spencer 1941). Hence, a pre-stressed anisotropic solid is a much realistic model to be used for seismic/elastic characterization of crustal rocks and composite materials.

A definitive theory explaining the elastodynamics of a body under initial stress was developed by Biot $(1939,1940)$. An elegant and elaborate exposition of this theory is found in Biot (1965). A large number of studies in the later years improved the understanding of wave propagation characteristics of materials under initial stress. Tolstoy (1982) studied the effect of gravity and hydrostatic pressure on velocities of elastic waves. Sharma and

Keywords. General anisotropy; initial stress; reflection. 
Gogna (1991) studied the effect of initial stress on Love waves in sandy layer over a poroelastic solid. Norris and Sinha (1995) studied the effects of anisotropy and pre-stress on the wave propagation along a fluid-solid interface. Degtyar and Rokhlin (1995, 1997) discussed the stress effects on ultrasonic wave velocities. In another study, Degtyar and Rokhlin (1998) discussed the effect of initial stress on wave propagation through an anisotropic/anisotropic interface. They used the stress dependent orthotropic elastic coefficients from a study by Man and Lu (1987). Surface wave velocities in a pre-stressed anisotropic solid is determined by Chai and $\mathrm{Wu}$ (1996). In a recent study, Prikazchikov and Rogerson (2003) analyzed the dynamic properties of anisotropic pre-stressed elastic solids. More recently, the effects of initial stress are studied on the velocities of three waves in general anisotropic elastic medium (Sharma and Garg 2006) and anisotropic poroelastic medium (Sharma 2005). The applications of the studies to the earth-system were also discussed.

In the present study, a procedure is explained to analyze the effects of initial stress on the reflection phenomenon in a general anisotropic elastic medium. The incidence of each of the three quasiwaves ( $q P, q S 1, q S 2$; Crampin 1981) is considered at the stress-free plane surface of the medium. Numerical model of Dolomite is used to compute the effects of initial stress on the phase directions, attenuations and reflection coefficients of the reflected waves. The Carbon-epoxy is used as a numerical model to explain the reflection of $q S 1$ waves in a medium with transverse isotropy.

\section{Definition of the problem}

The problem is to study the wave propagation in a general anisotropic solid half-space in the presence of initial stress. Three quasi-waves propagate in this medium. Incidence of a homogeneous wave along a given phase direction (arbitrary in 3-D space) is considered at the free surface of this nondissipative medium. The properties of three waves reflecting back into the medium are explored as follows:

- The Christoffel equations are solved algebraically, for phase velocities of the three quasiwaves as roots of a cubic equation. The waves $(q P, q S 1, q S 2)$ are identified with their velocity expressions (Appendix A).

- The Christoffel equations are solved into a polynomial equation of degree six. The numerically obtained six roots of this equation define the vertical slowness values for the three up-going and three down-going waves in the medium (Keith and Crampin 1977).
- Out of the six vertical slowness values, three are sorted out to represent the (reflected) waves traveling in the medium but away from its boundary.

- The types of the reflected waves corresponding to these three slowness values are identified using their velocity expressions.

- The slowness vectors of the reflected waves are resolved to calculate their propagation and attenuation characteristics.

- Energy partition is calculated at the free surface to find the reflection coefficients of the reflected waves.

- The numerical examples are considered to compute the velocities, attenuation and energy shares of reflected waves in the presence/absence of initial stress.

\section{Initially-stressed anisotropic medium}

\subsection{Equation of motion}

Consider a general anisotropic elastic medium under homogeneous initial stress $S_{i j}$. Following Biot (1965), the constitutive equations for wave motion in this medium, in the absence of body force, are given by

$$
s_{i j, j}+S_{j k} \omega_{i k, j}+S_{i k} \omega_{j k, j}=\rho \ddot{u}_{i},
$$

where $u_{i}$ are displacement components. The components of rotation are given by $\omega_{i j}=$ $\left(u_{i, j}-u_{j, i}\right) / 2$. Indices can take values of 1,2 , 3. The comma before an index represents (partial) space differentiation and dot denotes (partial) time derivative. Repeated index implies summation. The incremental stresses $s_{i j}$ in the medium are expressed as

$$
s_{i j}=B_{i j k l} u_{k, l},
$$

where the fourth rank asymmetric tensor $B_{i j k l}$ $\left(=B_{j i k l}=B_{i j l k}\right)$ represents the elastic properties of the medium. $\rho$ is density of the medium. Another property given by

$$
B_{i j k l}-B_{k l i j}=S_{k l} \delta_{i j}-S_{i j} \delta_{k l},
$$

ensures the existence of energy density function for the medium (Biot 1965), where $\delta_{i j}$ is Kronecker delta.

\subsection{Plane wave propagation}

To seek the harmonic solution of (1) for the propagation of plane waves, write

$$
u_{j}=U_{j} \exp \left\{\imath \omega\left(p_{k} x_{k}-t\right)\right\}, \quad(j=1,2,3),
$$


where $\omega$ is frequency and $\left(p_{1}, p_{2}, p_{3}\right)$ is slowness vector. Substituting (4) in (1) yields a system of three homogeneous equations, given by

$$
\left[Y_{i k}(\mathbf{p}, \mathbf{p})-\rho \delta_{i k}\right] U_{k}=0, \quad(i=1,2,3)
$$

where $\mathbf{p}$ denotes the slowness vector. $\mathbf{Y}$, a symmetric square matrix of order three, is defined in Appendix B. The system (5) may be called the modified Christoffel equations for pre-stressed anisotropic elastic medium. Non-trivial solution of this system is ensured by the determinantal equation

$$
\operatorname{det}[\mathbf{Y}(\mathbf{p}, \mathbf{p})-\rho \mathbf{I}]=0
$$

For the propagation of homogeneous plane waves in the medium, write $p_{j}=n_{j} / v ;(j=1,2,3)$, so that $v$ is the phase velocity of a wave along the phase direction $\left(n_{1}, n_{2}, n_{3}\right)$. Then, the system (5) defines a third order eigensystem (Appendix A) and its three eigenvalues explain the propagation of three quasi-waves $(q P, q S 1, q S 2)$ in the medium. The expressions for phase velocities $\left(v_{j} ; j=1,2,3\right)$ of these waves are derived in Appendix A. The normalised eigenvectors of system (5), i.e., $\left(U_{1}, U_{2}, U_{3}\right)$, define the polarizations of waves in the medium. The mathematical model derived so far is enough only to explain the propagation of three waves in an unbounded anisotropic elastic medium in the presence of initial stress.

\section{Reflection}

In most general cases, six waves are generated by the existence of a boundary in anisotropic elastic medium (Rokhlin et al 1986). The Snell's Law for such a medium ensures that the phase directions of all these waves lie in the same vertical plane, i.e., $\left(p_{1}, p_{2}\right)$ is same for all the waves. The six waves differ in vertical component (i.e., $p_{3}$ ) of slowness vector. So, for a given incident wave, $p_{1}$ and $p_{2}$ are known but the vertical slowness $p_{3}$ of a wave at the boundary is treated as an unknown, say $q$. For the slowness vector $\mathbf{p}=\left(p_{1}, p_{2}, q\right)$, the equation (6) is solved as a polynomial of degree six in $q$, given by

$$
\sum_{n=0}^{6} \alpha_{n} q^{n}=0 .
$$

The coefficients of this polynomial equation are derived in Appendix B. The roots of this equation are obtained numerically using the relevant functions or routines available in some computer softwares (e.g., MATLAB). The six roots of this

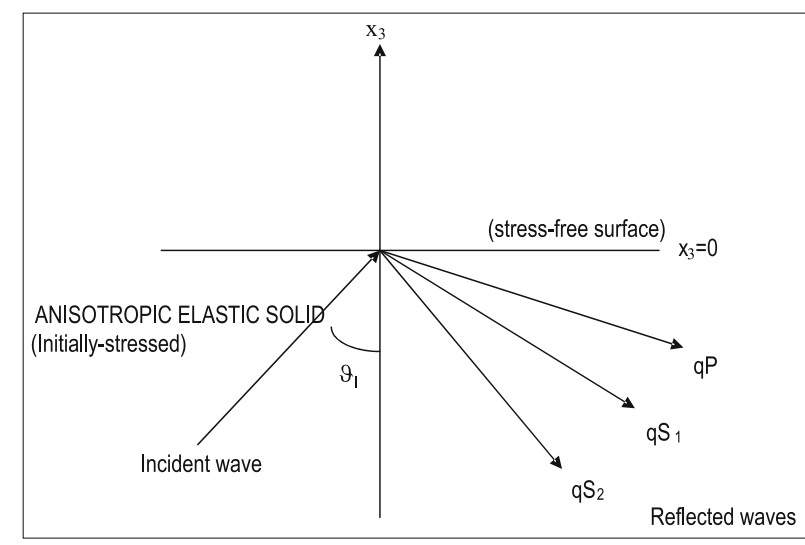

Figure 1. Geometry of the medium (plane $\phi_{I}=\pi / 4$ ).

equation explain the upward (positive $x_{3}$-direction) and downward (negative $x_{3}$-direction) propagation of three quasi-waves in the medium. For propagation in anisotropic solid layer all the six slowness values $(q)$ are required to define the displacement but for propagation in a half-space only three of them are physically acceptable.

\subsection{Geometry of the medium}

Consider an anisotropic elastic half-space, in a rectangular co-ordinate system $\left(x_{1}, x_{2}, x_{3}\right)$ under the homogeneous initial stress $S_{i j}$. The plane $x_{3}=0$ is the surface of this medium with $x_{3}$-axis, its outward normal (figure 1). For a general direction $(\theta, \phi)$ in the right hand co-ordinate system $\left(x_{1}, x_{2}, x_{3}\right), \theta$ is the polar angle with $x_{3}$-axis and $\phi$ is azimuth in $x_{1}-x_{2}$ plane, measured from $x_{1}$-axis to $x_{2}$-axis. A plane harmonic wave travels through this medium and becomes incident at a point on the surface. Let $\left(\theta_{I}, \phi_{I}\right)$ be the phase direction of the incident wave and it is used to define a unit vector $\mathbf{N}=\left(\sin \theta_{I} \cos \phi_{I}, \quad \sin \theta_{I} \sin \phi_{I}, \quad \cos \theta_{I}\right)$. This $\mathbf{N}$ is used to calculate the velocity (Appendix A) $v_{0}$ of the incident wave. This provides $p_{j}=N_{j} / v_{o},(j=$ $1,2)$, and vertical slowness of the incident wave is given by $q_{o}=N_{3} / v_{o}$. In this study, the medium is non-dissipative and, hence the incident wave is a homogeneous wave, i.e., $p_{1}, p_{2}$ are real. These values of $p_{1}$ and $p_{2}$ are used to calculate the six values of $q$ from (7).

The incidence through the medium results in the three waves reflected back into the medium, each with its own slowness vector $\left(p_{1}, p_{2}, q\right)$. The slowness vectors of these reflected waves are resolved to calculate their propagation and attenuation parameters. A vector $\left(p_{1}, p_{2}, \operatorname{Re}\{q\}\right)$ defines the propagation direction $(\theta)$ and inverse of propagation velocity $(c)$ of the wave. Another vector $(0,0, \operatorname{Im}\{q\})$ provides its attenuation $(\chi=\operatorname{Re}\{q\})$ along $x_{3}$ direction. 


\subsection{Slowness of reflected waves}

Out of these six values of $q$ corresponding to the $\left(p_{1}, p_{2}\right)$ of incident wave, the three generally represent the reflected waves. One of the remaining three will be equal to $q_{o}$ and hence represents the incident wave. This happens for the incidence of each of the three types of waves in the medium. Note that this is a self-check to ensure the correctness of the analytical expressions as well as the numerical implementation. Synge (1957) has mentioned the procedure to choose the physically acceptable vertical slowness values to represent reflected waves. In the present geometry, a complex vertical slowness $(q)$ with negative imaginary part ensures that the (reflected) wave will be decaying on moving away from the surface.

A wave with real value of $q$ represents a reflected wave when its corresponding ray-direction is away from the boundary. The vector $\mathbf{N}$ and corresponding phase velocity $v$ are used to find the group velocity and ray direction of the reflected wave. The $x_{3}$-component of group velocity (Appendix A) explains the direction of flow of energy normal to the boundary. In the present geometry, a positive (negative) value of $x_{3}$-component of group velocity (i.e., $g_{3}$ in Appendix A) implies that the corresponding wave is traveling towards (away from) the surface. In this way, the slowness values corresponding to the reflected waves are identified. It is generally expected that for every incidence, there should be exactly three slowness values to explain three different $(q P, q S 1, q S 2)$ reflected waves.

\subsection{Reflected waves identification}

The section 4.2 provides the slowness values corresponding to the reflected waves. But, to associate each of the slowness values of reflected waves with a particular type $(q P$ or $q S 1$ or $q S 2)$ of wave is an important task. In case of general anisotropy, the six roots of equation (7) can only be obtained numerically. The order in which these numerical roots are obtained may not remain same for any two different computations. A procedure to associate these three roots to different reflected waves may be explained as follows.

For each value of $q$ (amongst those corresponding to reflected waves), the slowness vector $\mathbf{p}=\left(p_{1}, p_{2}, q\right)$ is written as $\mathbf{N} / v$ so that $\mathbf{N} \cdot \mathbf{N}=1$. For this $\mathbf{N}$, the phase velocities $\left(v_{j} ; j=\right.$ $1,2,3)$ of the three waves in the medium are calculated from cubic equation (20) in Appendix A. Then, one of these three phase velocities must be equal to $v$. This identifies the slowness vector $\mathbf{p}$ with a particular wave. It may be noted that this certifies the correctness of the whole procedure solving the mathematical model.

\subsection{Reflection coefficients}

The displacement components in the anisotropic elastic medium are expressed as

$$
\begin{aligned}
u_{j} & =U_{j}^{(o)} \exp \left\{\imath \omega\left(p_{1} x_{1}+p_{2} x_{2}+q_{o} x_{3}-t\right)\right\} \\
& +\sum_{m=1}^{3} f_{m} U_{j}^{(m)} \exp \left\{\imath \omega\left(p_{1} x_{1}+p_{2} x_{2}+q_{m} x_{3}-t\right)\right\},
\end{aligned}
$$

where $f_{m}$ are relative excitation factors. Product of $f_{m}$ with polarisation component $U_{j}^{(m)}$ defines the contribution of wave ' $m$ ' to the magnitude of $j$ th component of displacement.

The equations (2) relate stresses to displacement components $u_{i}$. On a unit elementary area of initial boundary $\left(x_{3}=0\right)$, the incremental boundary force, after deformation, is given by

$$
\begin{aligned}
\Delta F_{i}= & s_{i 3}+S_{k 3} \omega_{i k}+S_{i 3} e_{k k}-S_{i k} e_{3 k}, \\
& (i=1,2,3) .
\end{aligned}
$$

The boundary conditions appropriate for the free surface are the vanishing of incremental boundary forces. So, the three boundary conditions required to be satisfied at the plane $x_{3}=0$, are

$$
\Delta F_{i}=0, \quad(i=1,2,3) .
$$

Satisfying the above boundary conditions yields a system of three linear inhomogeneous equations in $f_{1}, f_{2}$ and $f_{3}$. These equations are given by

$$
a_{j k} f_{k}=b_{j} ; \quad(j=1,2,3),
$$

where for vectors $\mathbf{p}^{(\mathbf{k})}=\left(p_{1}, p_{2}, q_{k}\right)^{T}$, and $\mathbf{U}^{(\mathbf{k})}=$ $\left(U_{1}^{(k)}, U_{2}^{(k)}, U_{3}^{(k)}\right)$, the coefficients of system (11) are expressed as

$$
\begin{aligned}
a_{j k}= & \left(\mathbf{U}^{(k)} \otimes \mathbf{p}^{(k)}\right) \cdot\left(\mathbf{B}^{(j)}+\boldsymbol{\Sigma}^{(j)}\right) ; \\
& (j, k=1,2,3),
\end{aligned}
$$

where ' $\otimes$ ' and '.' denote the tensor product and dot product between two tensors, respectively. Similarly, $b_{j}=-\left(\mathbf{U}^{(o)} \otimes \mathbf{p}^{(o)}\right) \cdot\left(\mathbf{B}^{(j)}+\boldsymbol{\Sigma}^{(j)}\right),(j=1,2,3)$, where $\mathbf{U}^{(o)}$ and $\mathbf{p}^{(o)}$ are the polarization and slowness, respectively, of incident $q P$ or $q S 1$ or $q S 2$ waves. The second order tensors $\mathbf{B}^{(j)}$ and $\boldsymbol{\Sigma}^{(j)}$ are defined as follows:

$$
\begin{aligned}
B^{(j)}= & \left\{b_{l 1}, b_{l 6}, b_{l 5} ; b_{l 6}, b_{l 2}, b_{l 4} ; b_{l 5}, b_{l 4}, b_{l 3}\right\} \\
& (l=6-j, \quad j=1,2,3)
\end{aligned}
$$


$\Sigma_{i k}^{(j)}=S_{j 3} \delta_{i k}+\frac{\left(S_{k 3} \delta_{i j}-S_{i j} \delta_{k 3}-S_{j k} \delta_{i 3}-S_{i 3} \delta_{j k}\right)}{2} ;$

$$
(i, k=1,2,3) .
$$

Reflection coefficient is the energy share of a reflected wave in the energy of the incident wave at the reflecting boundary. The rate at which the energy is communicated across the unit area of a surface is the scalar product of boundary force and particle velocity. Its average over a time period, denoted by $\langle P\rangle$, represents the average energy transmission per unit surface area per unit time. A matrix of energy ratios $E_{j k}=$ $\left\langle P_{j k}\right\rangle /\left\langle P_{I I}\right\rangle ;(j, k=1,2,3)$, is defined to explain the energy partition among the waves present in the anisotropic medium. $x_{3}$-axis being the outer normal to the surface, the $\left\langle P_{j k}\right\rangle$ representing the average energy fluxes, are given by

$$
\left\langle P_{j k}\right\rangle=0.5 \operatorname{Re}\left\{\Delta F_{i}^{(j)} \operatorname{conj}\left(\dot{u}_{i}^{(k)}\right)\right\}, \quad(j, k=1,2,3) .
$$

The diagonal entries of the energy matrix $\mathbf{E}$ represent the reflection coefficients of the three reflected waves. The sum of all the non-diagonal entries of this energy matrix gives the share of interaction energy (Borcherdt 1977) for the reflection. At incidence before all the critical angles, the off-diagonal part of this energy matrix is skew symmetric and hence, no interaction energy. At incidence beyond the critical angle for a reflected wave, the corresponding wave become inhomogeneous but offdiagonal elements of the energy matrix remain skew-symmetric. Conservation of energy is given by the relation $\sum_{j=1}^{3}\left(\sum_{k=1}^{3} E_{j k}\right)=1$.

\section{Numerical example}

The purpose of numerical computation is to analyze the effects of initial stress on the propagation of waves reflecting from the stress-free plane surface of a general anisotropic medium. The relevant propagation characteristics are phase velocity, phase direction, attenuation and reflection coefficient of each of the reflected waves. The general anisotropy is assumed in a Dolomite rock of density $2423 \mathrm{~kg} / \mathrm{m}^{3}$. The elastic matrix (in GPa) for Dolomite (Rasolofosaon and Zinszner 2002) is given by

$$
\begin{array}{lll}
b_{11}=65.53 & b_{12}=9.77 & b_{13}=12.19 \\
b_{14}=0.18 Z_{1} & b_{15}=-0.81 Z_{1} & b_{16}=2.94 Z_{2} ; \\
b_{22}=50.77 & b_{23}=11.61 & b_{24}=-0.09 Z_{1}
\end{array}
$$

$$
\begin{aligned}
& b_{25}=-0.50 Z_{1} \quad b_{26}=-0.19 Z_{2} \\
& b_{33}=60.11 \quad b_{34}=-1.61 Z_{1} \\
& b_{35}=1.78 Z_{1} \quad b_{36}=0.84 Z_{2} ; \\
& b_{44}=23.51 \quad b_{45}=1.49 Z_{2} \quad b_{46}=-1.17 Z_{1} \\
& b_{55}=24.57 \quad b_{56}=0.26 Z_{1} \quad b_{66}=20.21 .
\end{aligned}
$$

$b_{i j}$ are the components of square matrix of order six and represent the elastic constant tensor $B_{i j k l}$ in two-suffix notation. The matrix $\mathbf{b}$ is not a symmetric one and the remaining elements of the matrix are defined through the relations (3). The assumed values in matrix $\mathbf{S}=\left\{10,1.2 Z_{2}, 0.8 Z_{1}\right.$; $\left.1.2 Z_{2}, 9, Z_{1} ; 0.8 Z_{2}, Z_{1}, 11\right\}$, defines the amount (in GPa) of initial stress in the medium. The values of $Z_{1}=Z_{2}=1$ define the anisotropy of arbitrary (triclinic) type. The values $Z_{1}=0, Z_{2}=1$ represent the monoclinic symmetry and $Z_{1}=Z_{2}=0$ represent the orthorhombic symmetry, in the elastic tensor as well as initial-stress tensor.

The incidence of each of the $q P, q S 1$ and $q S 2$ waves is considered. The incident wave is restricted to a vertical plane, fixed by $\phi=0.25 \pi$. This defines a plane of propagation for all the waves in the medium. In the propagation plane, the phase direction $\theta_{I}$, of the incident wave varies from 0 to $90^{\circ}$. The slowness of each reflected wave is resolved for propagation velocity $(c)$, propagation direction $(\theta)$. The non-zero value of attenuation factor $(\chi)$ is obtained when the concerned reflected wave becomes inhomogeneous. However, for incidence of a homogeneous wave, the attenuation directions of the reflected waves are always normal to the surface. The energy matrix is computed to calculate the energy ratios of reflected waves and the interaction energy.

\section{Discussion of numerical results}

Figure 2 exhibits the variations of $c, \theta, \chi$ and the reflection coefficients of the reflected waves resulting from the incidence of $q P$ waves. The medium is anisotropic with arbitrary symmetry (i.e., triclinic). It may be noted that all the reflected waves are homogeneous and hence no attenuation. The presence of initial stress results in a very small deviation in reflected waves towards the surface. But the effects of initial stress on the phase velocities and reflection coefficients of the three reflected waves are quite clear and significant. The velocity and energy share of $q S 1(q S 2)$ waves decrease (increase) with the presence of initial stress. The 

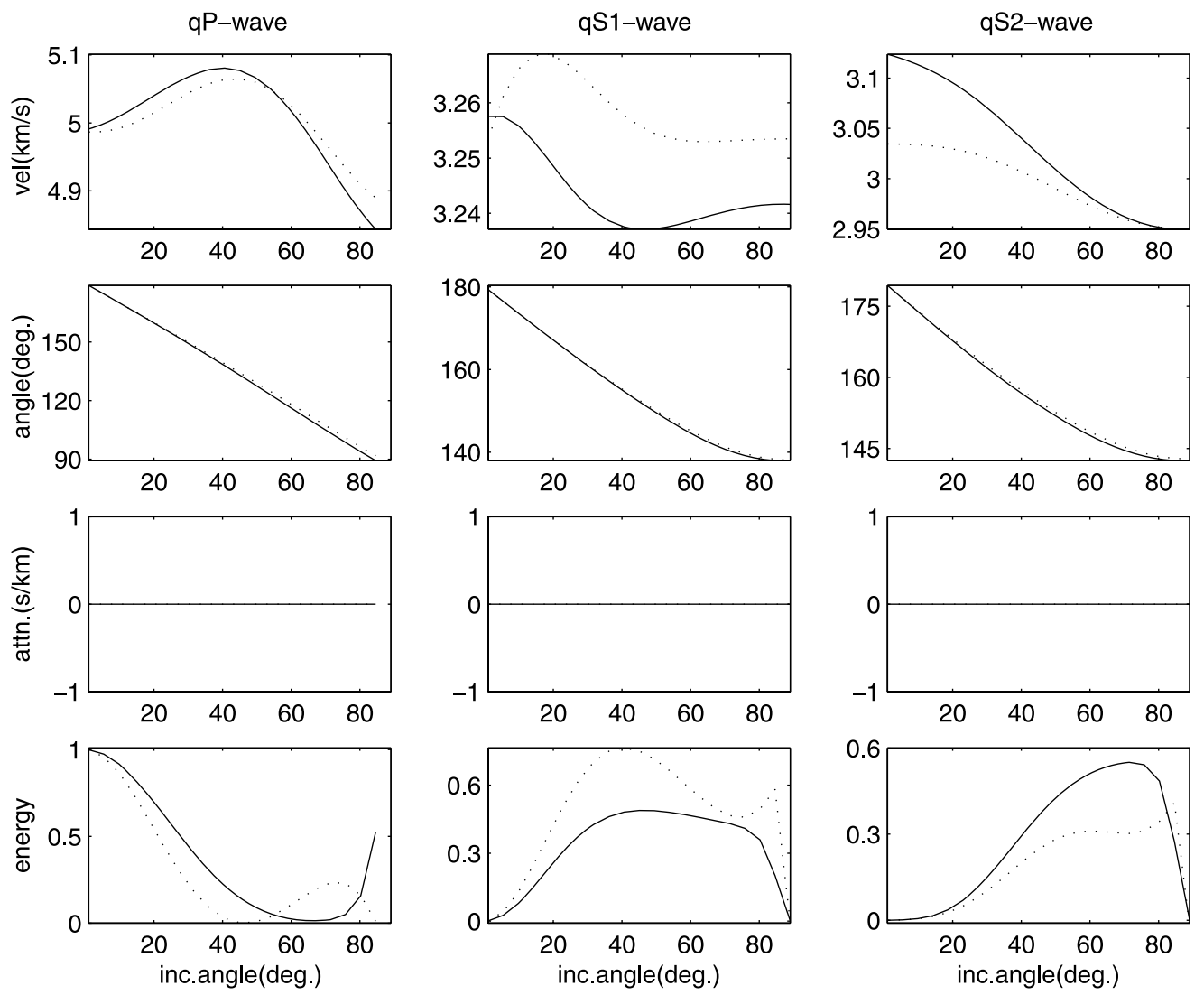

Figure 2. Phase velocity $(c)$, phase angle $(\theta)$, attenuation factor $(\chi)$ and energy share $(E)$ of three reflected waves $(q P, q S 1, q S 2)$ in Dolomite rock with triclinic (TCS) anisotropy; solid (dotted) curves for presence (absence) of initial stress; incident $q P$ wave.

presence of initial stress increases the velocity and reflection coefficient of $q P$ wave but for incidence up to a certain angle (nearly $50^{\circ}$ ) and reverses beyond that. That means there may be an incident direction for $q P$ waves along which the velocity and energy of the reflected $q P$ wave is not affected by the presence of initial stress. Similar directions for reflected $q S 1$ and $q S 2$ waves are the normal and grazing incidence, respectively. The figure 3 presents the response of reflected waves from the incident $q P$ waves, to the initial stress in the elastic media with monoclinic anisotropy. The presence of a symmetry, may have a little effect on the reflection coefficients but have a significant effect on the velocities of the reflected waves. Now the presence of initial stress decreases the velocity of reflected $q P$ waves but increases the velocities of the other two slower waves. Moreover, for normal incidence of $q P$ waves, the reflected $q P$ waves are unaffected by the presence of initial stress. With the presence of another symmetry, i.e., orthorhombic anisotropy (figure 4), the energy share of reflected $q P$ wave is affected slightly, but the reflected coefficient of $q S 1$ waves is reduced nearly to half. But the loss of $q S 1$ waves becomes the gain of $q S 2$ waves. The velocities of reflected waves are affected with the presence of anisotropic symmetry as well as initial stress. For example, the effect on initial stress on the reflection coefficient of the fastest $(q P)$ wave is opposite to that on the other two waves. However, the response of two slower ( $q S 1$ and $q S 2)$ waves to the initial stress is nearly similar. The velocity of reflected $q P$ waves may not be affected by the initial stress when the incidence is normal or grazing.

Figure 5 contains the propagation characteristics of the reflected waves resulting from the incidence of $q S 1$ waves in a general (triclinic) anisotropic medium. Comparison of the plots in this figure to the corresponding plots in figure 2 explains the difference between the incidence of $q P$ waves and $q S 1$ waves. One of the major differences is the presence of the critical angle (nearly $40^{\circ}$ ) for reflected $q P$ wave and, then its propagation as inhomogeneous wave with a much-reduced velocity. Also the small effect of initial stress on the phase directions of reflected waves is reversed. Another major effect may be noted in the energy shares of all the reflected waves. The variations of the effect of initial stress on the reflection coefficients 

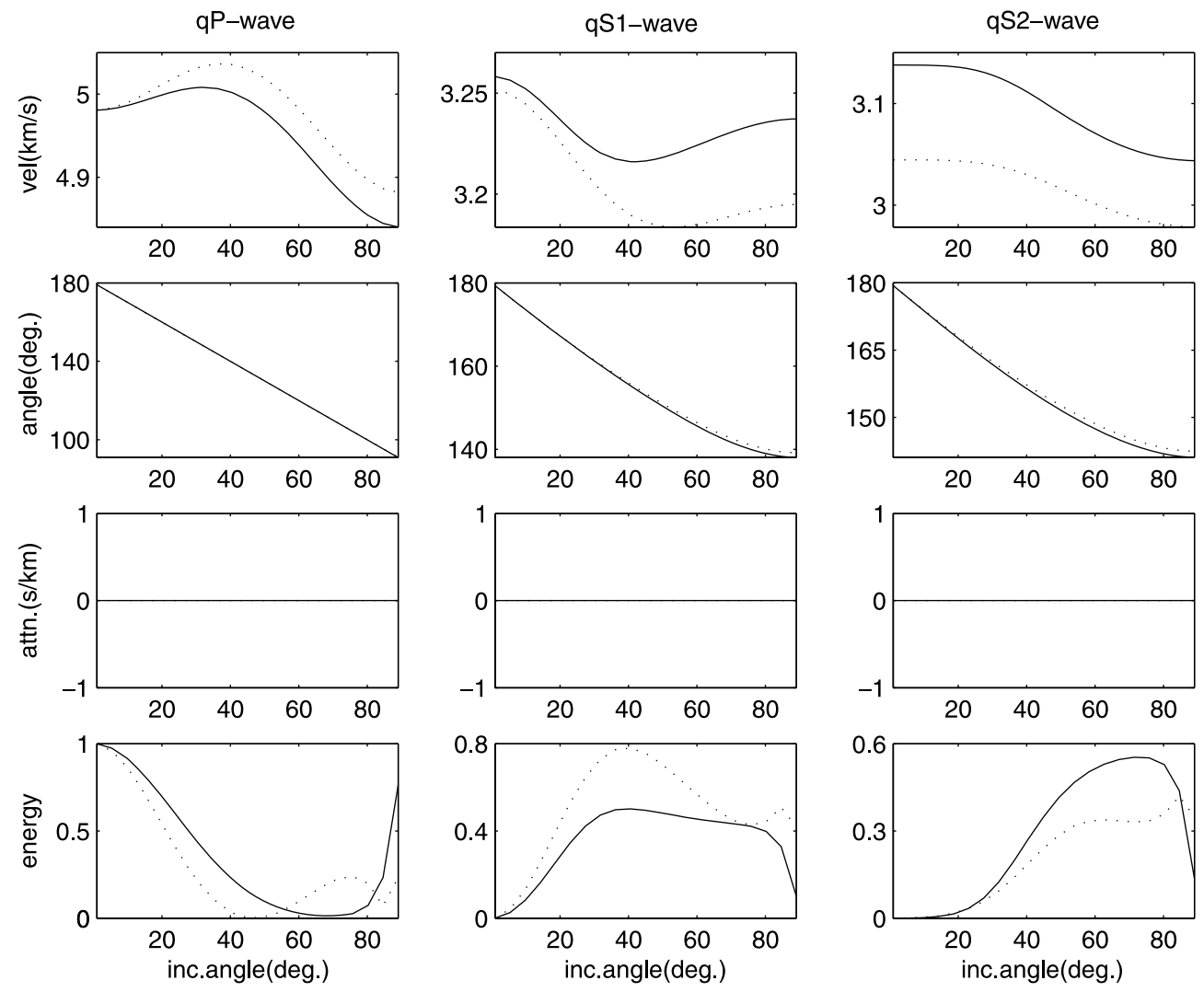

Figure 3. Same as figure 2, but for monoclinic (MCS) anisotropy.
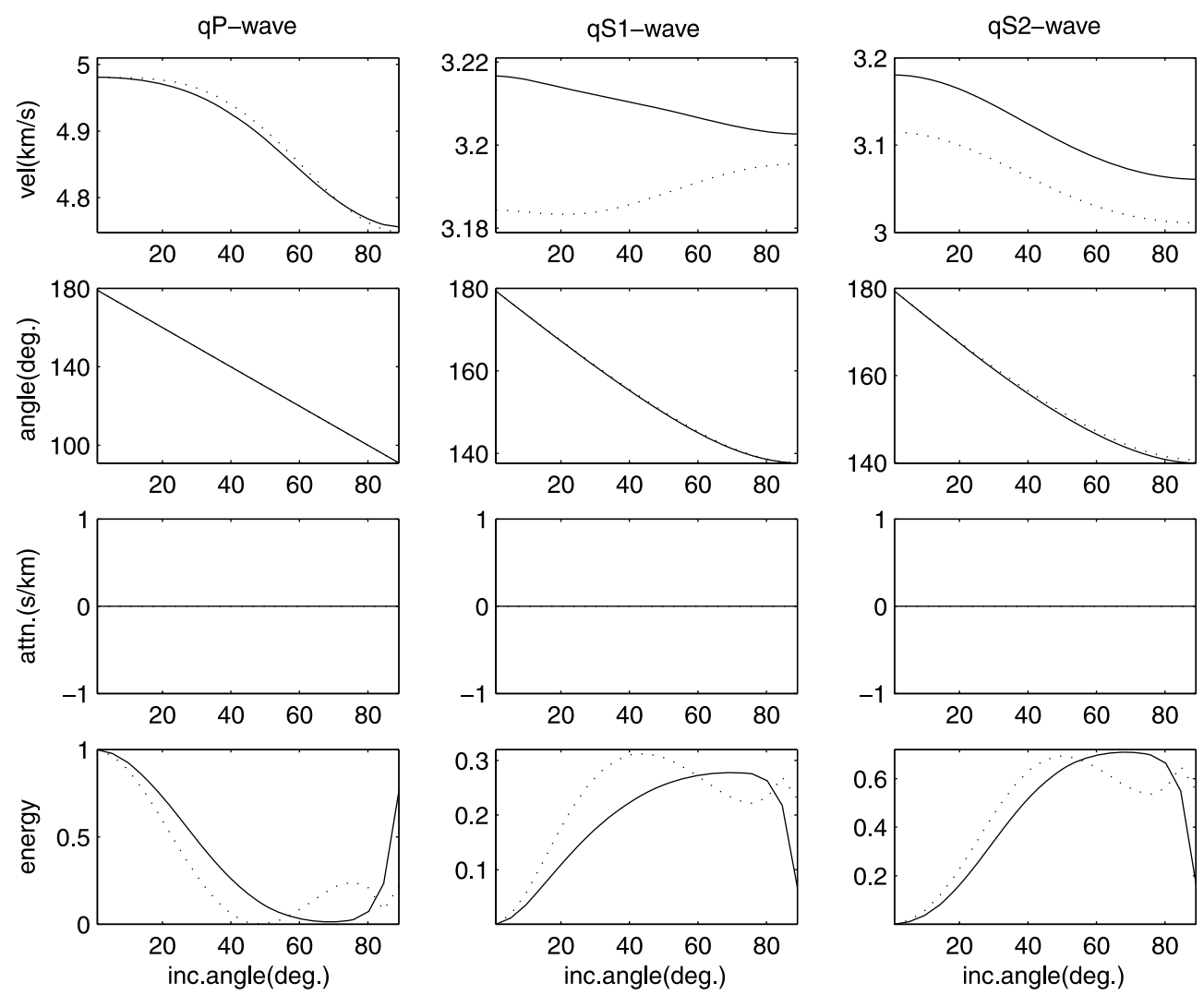

Figure 4. Same as figure 2, but for orthotropic (ORS) anisotropy. 

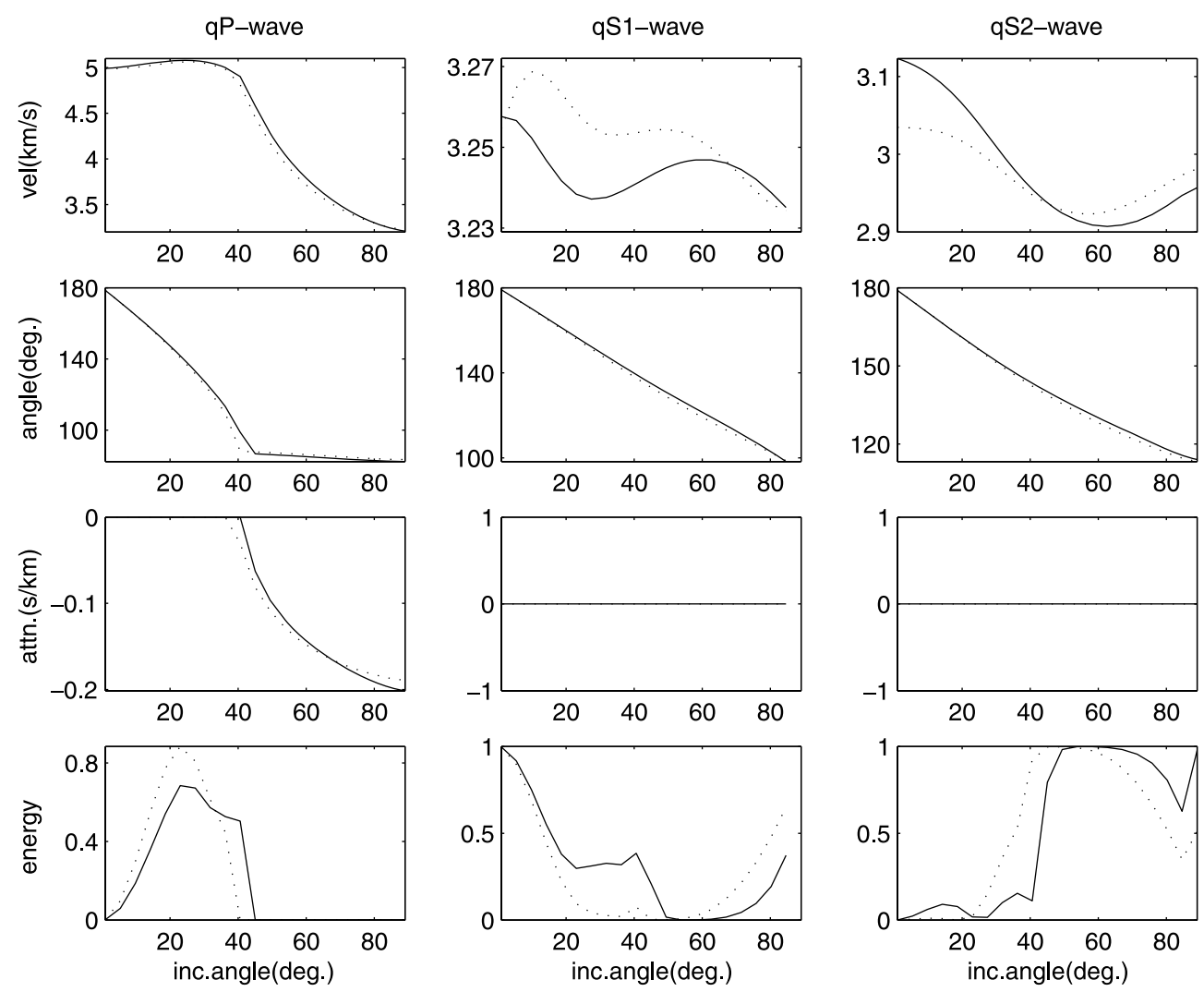

Figure 5. Same as figure 2, but for incident $q S 1$ wave.

with incident direction are completely different in both the figures. For the presence of (monoclinic) anisotropic symmetry, figure 6 explains the propagation of reflected waves resulting from the incident $q S 1$ waves. Similar to the incident $q P$ waves, the effects of initial stress are changed mainly on the velocities of $q S 1$ and $q S 2$ waves. The energy shares of the reflected waves are also affected with the presence of anisotropic symmetry. For the presence of another (orthorhombic) symmetry, figure 7 shows the effect of initial stress on the reflection phenomenon. The velocity variations of reflected $q S 1$ and $q S 2$ waves with the incident direction are not same as in figures 5 and 6 . The changes in reflection coefficients are observed but their variations with incident directions appear, nearly similar for the three different anisotropies.

For the incidence of $q S 2$ waves, figures 8 to 10 present the reflection phenomenon in anisotropic media with triclinic, monoclinic or orthorhombic symmetry, respectively. Comparing figure 8 with figure 5, it may be noticed that the reflected $q P$ waves are different only in energy. The effects of initial stress on this energy are opposite in two figures. The effect of initial stress on the propagation characteristics of the other two waves are very much different in these figures. For the incident $q S 2$ waves, the reflected $q S 1$ wave becomes evanescent for incidence beyond its critical angle. The effect of initial stress on the critical angle is quite significant. For incidence near the critical angle for reflected $q P$ waves, the reflected $q S 2$ waves get a jump in its energy share. Similarly, the presence of anisotropic symmetry may not affect reflected $q P$ waves much. However, the propagation characteristics of $q S 1$ and $q S 2$ waves are changing significantly with the anisotropy type.

\section{Transverse isotropy}

This study in a transversely isotropic medium may be an interesting case for geophysicists. In the presence of initial-stress, an isotropic or transversely isotropic medium behaves orthotropic to wave propagation. Hence, there is no problem in applying the model presented to the media with symmetries higher than orthorhombic. However, in the absence of initial stress, the propagation of $q S 2$ wave decouples (as $\mathrm{SH}$ ) from $q P$ to $q S 1$ waves in the transversely isotropic medium with vertical $\left(x_{3}\right)$ axis of symmetry (VTI). Then, the third order system in reflection phenomenon is broken into a system of order 2 and a linear system. But, this can be avoided by replacing a zero value in symmetric elastic matrix $\left(b_{I J}\right)$ with a very small 

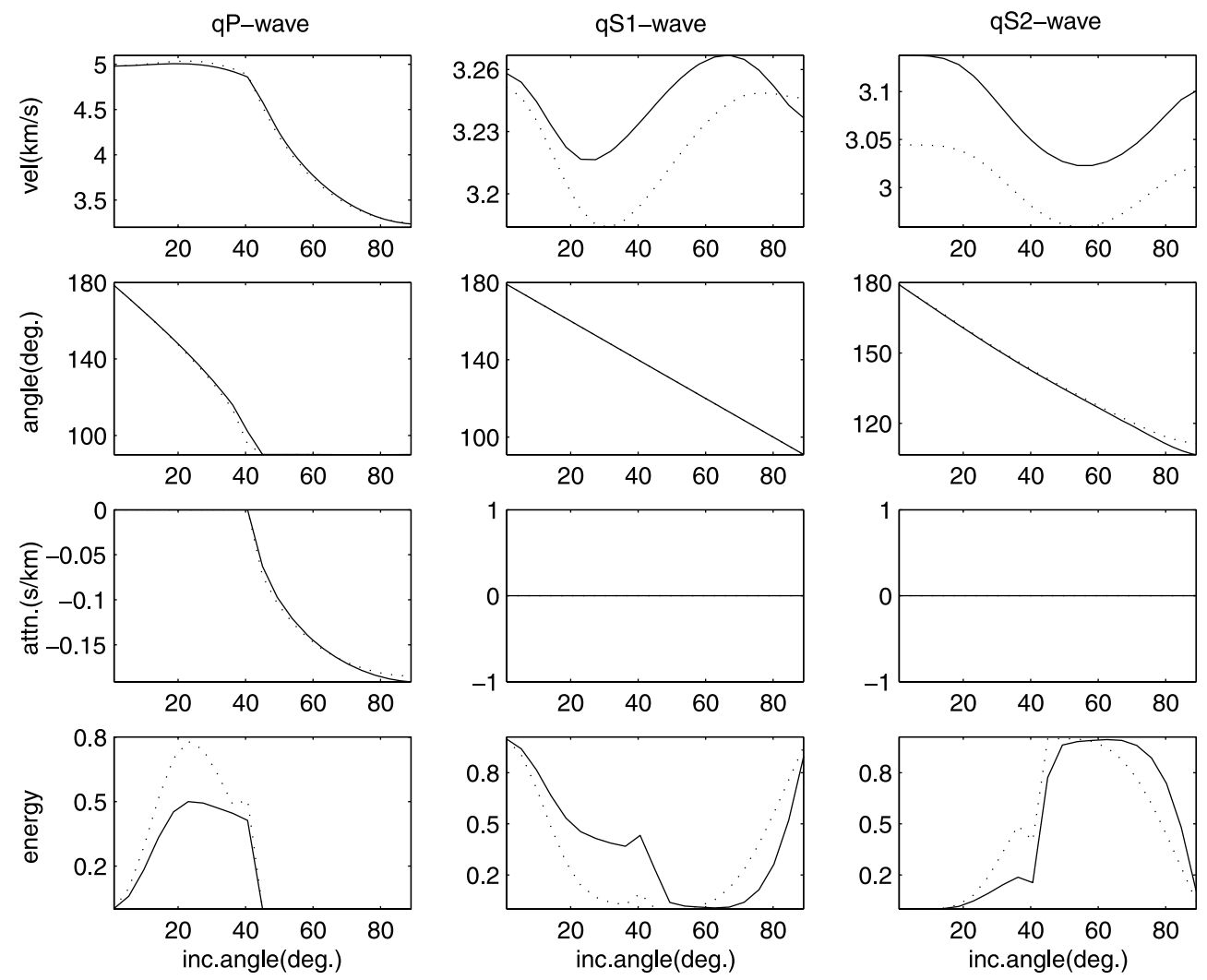

Figure 6. Same as figure 3, but for incident $q S 1$ wave.
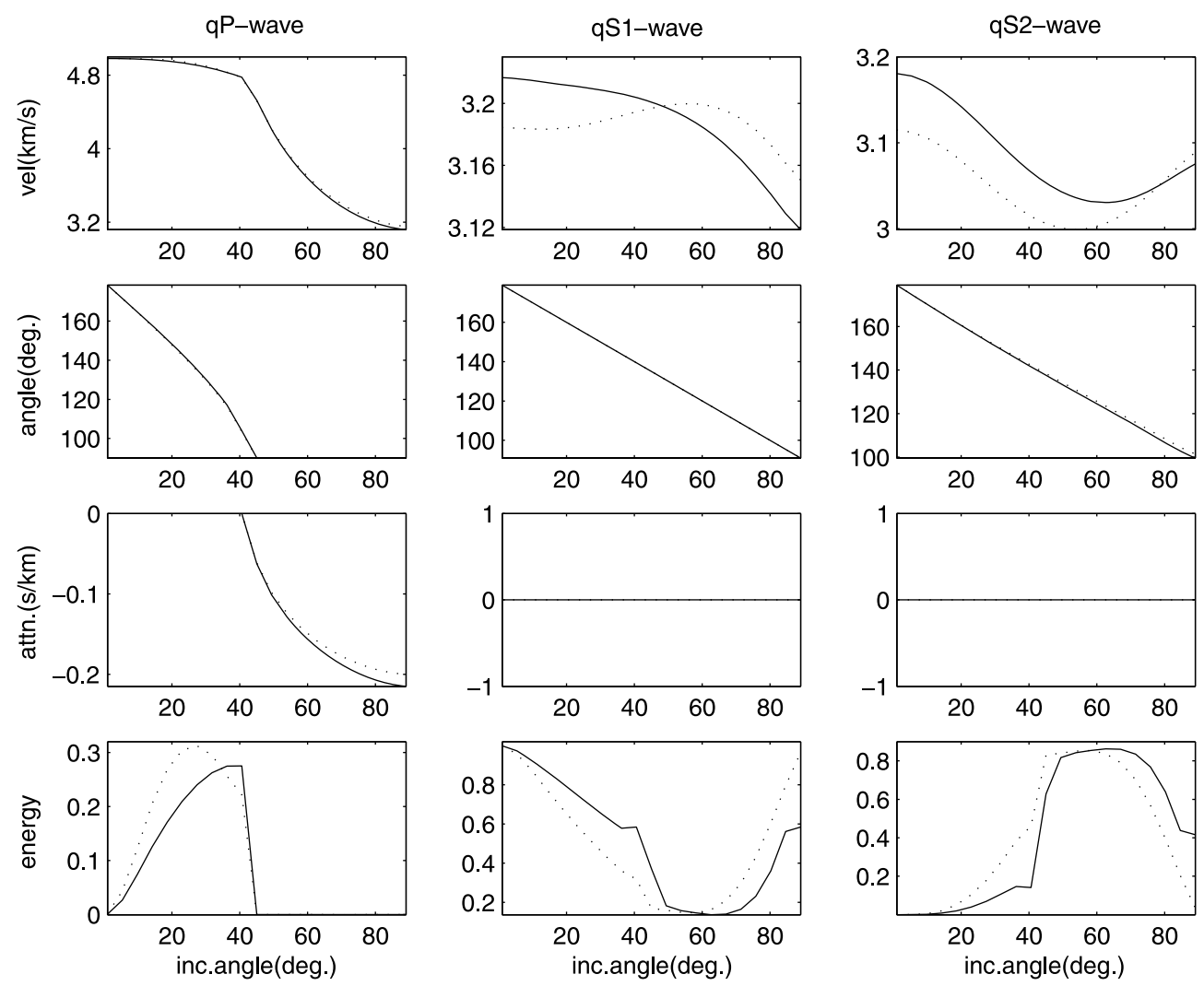

Figure 7. Same as figure 4, but for incident $q S 1$ wave. 

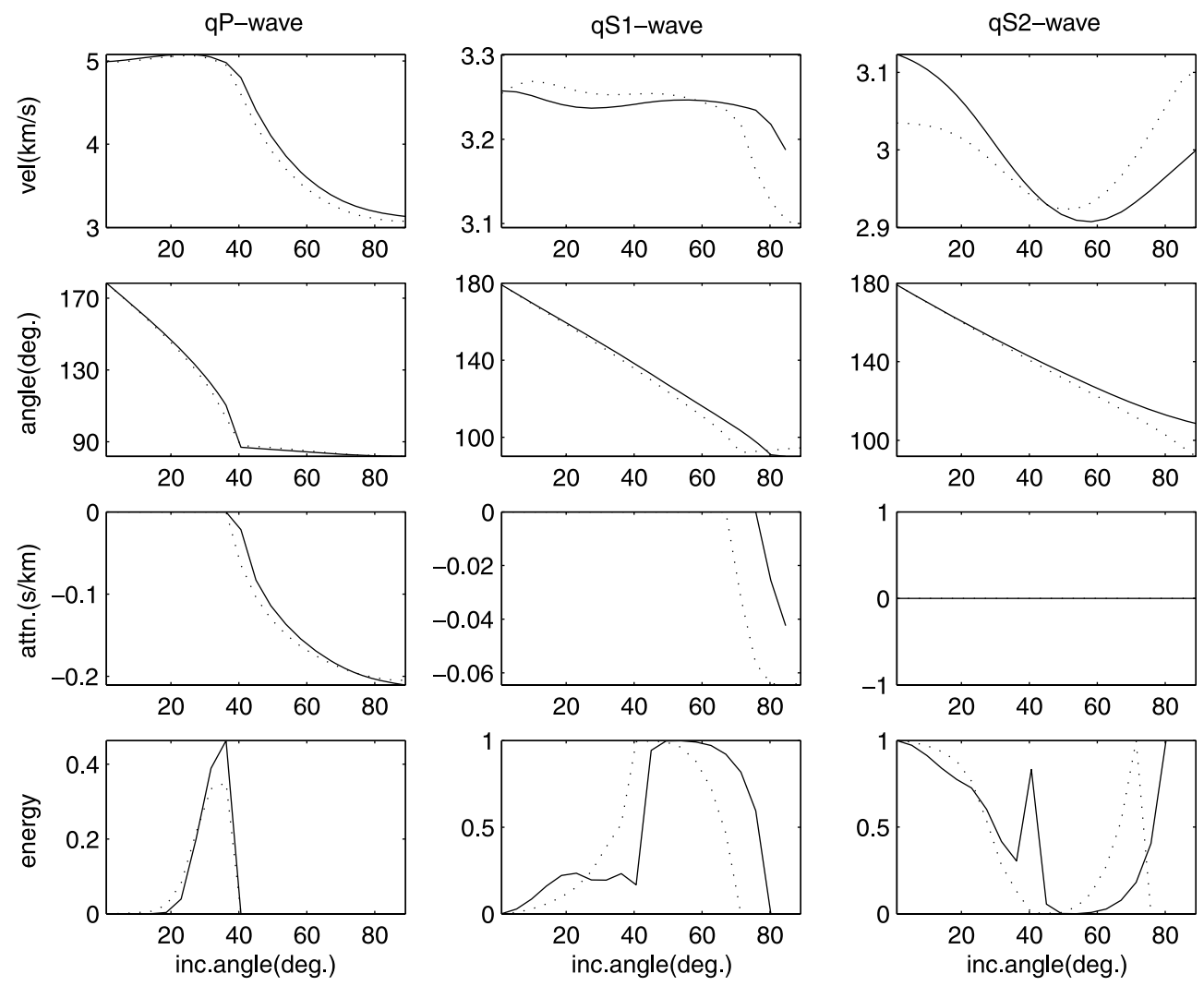

Figure 8. Same as figure 2, but for incident $q S 2$ wave.
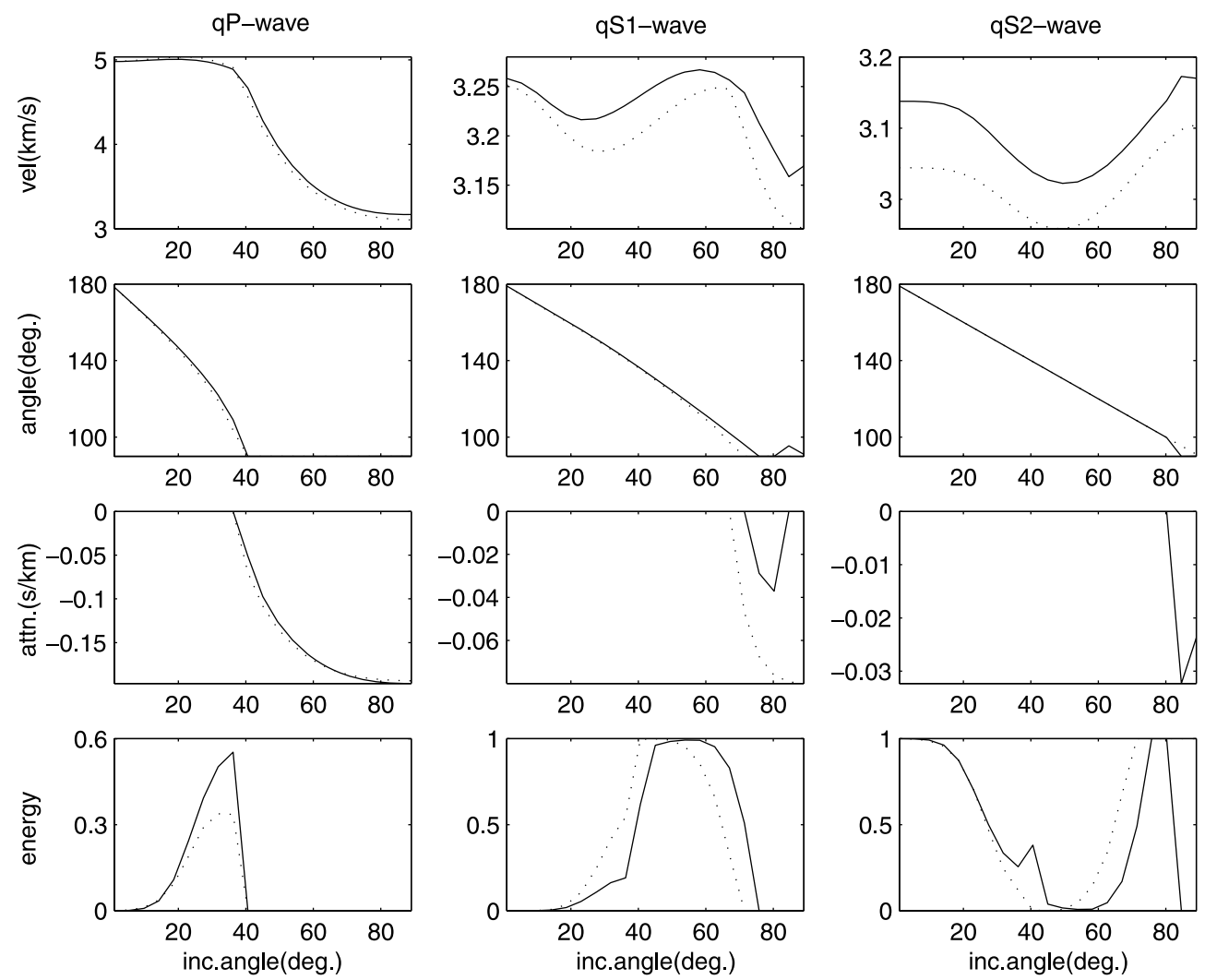

Figure 9. Same as figure 3, but for incident $q S 2$ wave. 

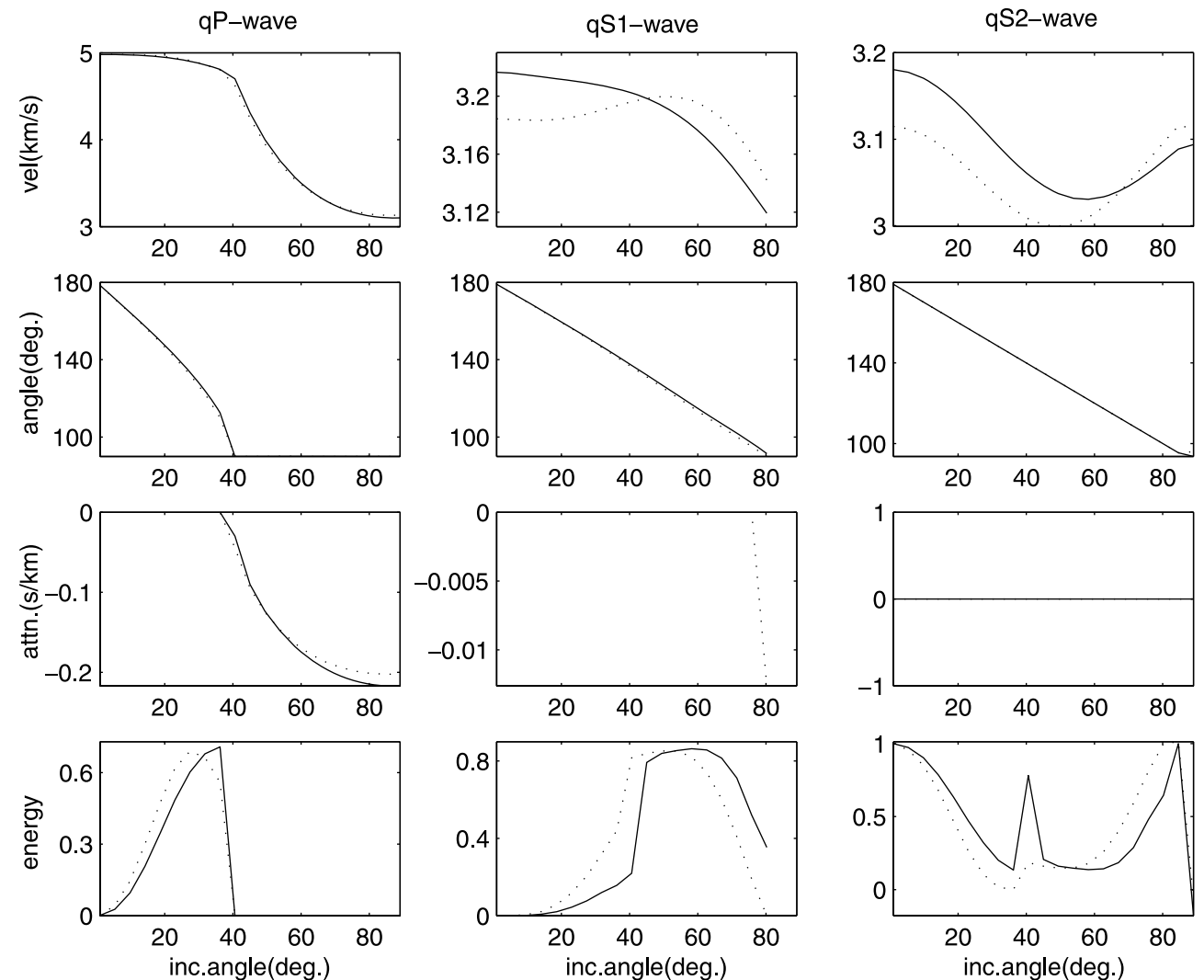

Figure 10. Same as figure 4, but for incident $q S 2$ wave.
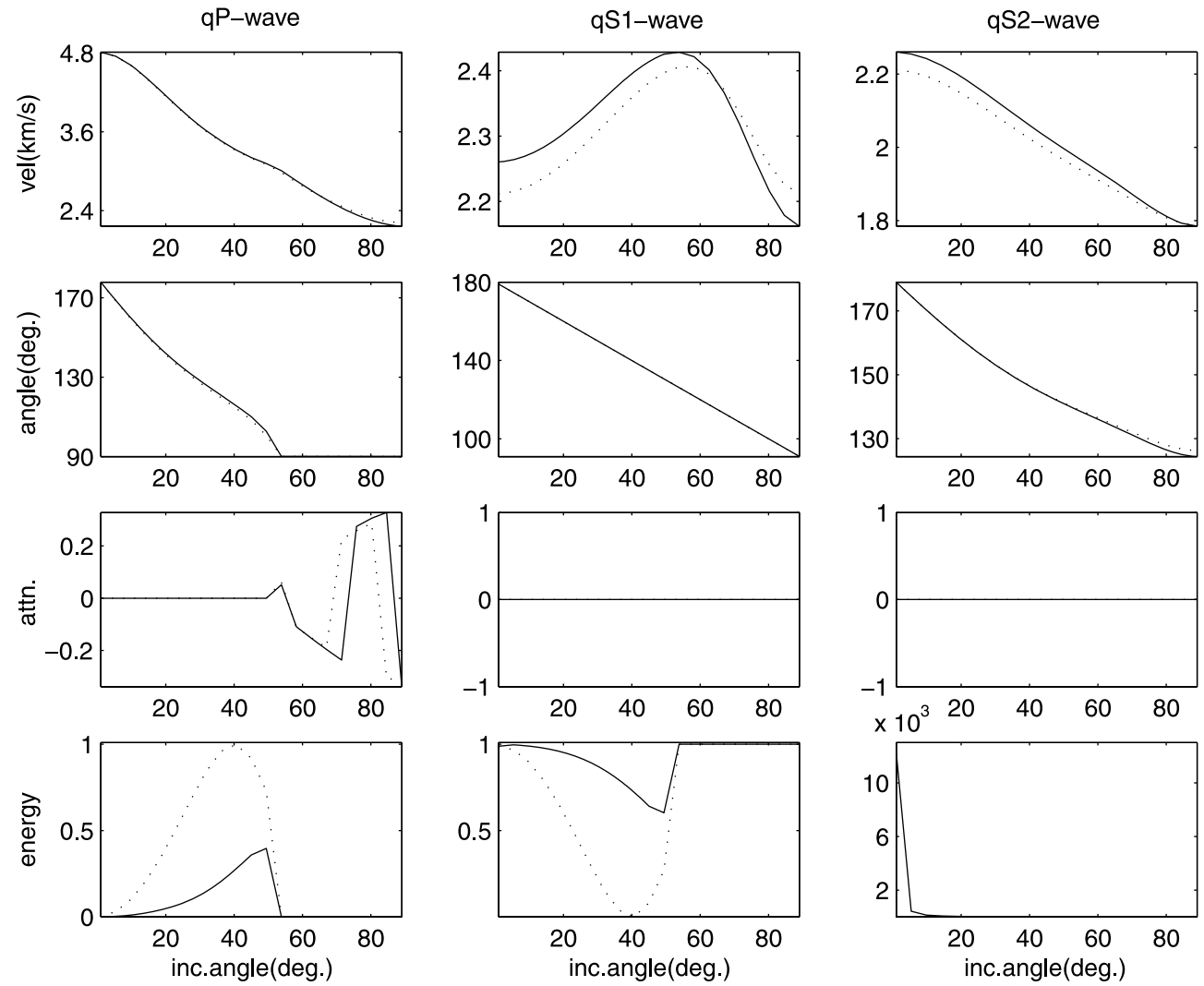

Figure 11. Same as figure 5 (i.e., incident $q S 1$ wave), but medium is Carbon-epoxy with transverse isotropy (VTI). 
non-zero value. Such a replacement may not have much effect on the propagation characteristics of the reflected waves. The incidence of $q S 1$ wave is considered in a numerical model of carbon-epoxy (a composite material made from carbon fibers plunged into an epoxy resin matrix) as VTI solid of density $1595 \mathrm{~kg} / \mathrm{m}^{3}$. The elastic moduli (Lanceleur et al 1993) are given by $\left(b_{11}, b_{12}, b_{13}, b_{33}, b_{44}\right)=$ $(15,7.7,3.4,37$, 7.8) GPa. The initial-stress is defined by $S_{11}=S_{22}=7 \mathrm{GPa}, S_{33}=7.7 \mathrm{GPa}$. The velocities, phase directions, critical angle and reflection coefficients of the reflected waves are as shown in figure 11 . The differences in corresponding plots of this figure with those in figure 7 are due to the change in elastic model as well as change in anisotropic symmetry. The major difference is that the energy share (reflection coefficient) of $q S 2$ wave is almost zero even in the presence of initial stress. This implies that the $q S 2$ is nearly decoupled for the value of initial stress chosen. However, the effect of initial stress is quite clear and significant on the reflected $q P$ and $q S 1$ waves.

\section{Conclusions}

The numerical example discussed above implies that the mathematical model solved in this work may be used to compute the exact effects of initial stress on propagation characteristics of the waves at the boundary of general anisotropic solid medium. The anisotropy in the model should be viewed as means of investigating the internal constitution of the rocks, materials, structures, etc. Initial stress in the model represents in situ stress distribution. The numerical results discussed in previous section are based on a particular numerical model and hence, may not qualify for quantitative generalisation. However, the qualitative interpretation of these results may help to extract the following few useful conclusions:

- The effect of initial stress is negligible on the phase directions of the reflected waves.

- The effect of initial stress is very small on the phase velocity of the reflected $q P$ waves, particularly from the incidence of $q S 1$ or $q S 2$ waves.

- In general, the effects of initial stress on the reflected waves vary with the direction of the incident wave, elastic properties and the anisotropy present in the medium.

- The critical angles and attenuations of the reflected $(q S 1, q S 2)$ waves change with the changes in elastic properties of the medium. The change in anisotropic symmetry may not have much effect on critical angles if the values of non-zero elastic moduli are unchanged.

- Energy partition among reflected waves is more sensitive to the presence of initial stress as compared to other propagation characteristics.

The importance of the work presented may be explained as follows:

- The mathematical model (equation 1) considers the wave propagation in a realistic medium which is more appropriate to represent in situ crustal materials and reservoir rocks.

- The mathematical model is solved (equation 7) for six slowness surfaces for the waves existing at a boundary of the medium. All the six slowness surfaces are required/used to define the displacement (particle motion) in a layered medium.

- For propagation in a half-space, the slowness surfaces of three outgoing waves (sections 4.2 and 4.3) are used to define displacement (and stress) there.

- The derived reflection procedure provides a platform to study a wave propagation problem in a multi-layered medium containing a pre-stressed anisotropic solid layer. A study on wave scattering from an inhomogeneity in the medium also requires this reflection procedure.

- The work presented relates the geophysical properties (anisotropy, initial-stress) of a realistic medium to its propagation characteristics (velocities, attenuation, polarizations, phase shift, reflection coefficients). When supported with a real data, such a relation may be used to understand a variety of geophysical problems, e.g.,

1) The characterization of residual stresses in the reservoirs, earth masses and composite materials.

2) The differential stress environment present in the saturated cracks which conducts the flow of fluid between the connected cracks in a reservoir.

3) The prediction of pre-drill overpressure, which is required for the monitoring of hydrocarbon production in the boreholes (Sayers et al 2002).

4) The information on fracture distribution (from velocity inversion) to estimate the anisotropic permeability of the fracture rock system (Gibson and Toksoz 1990).

5) The extent of fracturing in a region of a borehole, which is a vital factor in the extraction of oil and geothermal heat. 


\section{Appendix A: Velocities}

Consider a general anisotropic elastic medium represented by the elastic tensor $B_{i j k l}$ and density $\rho$. A non-symmetric square matrix of order six $\left\{b_{I J}\right\}$, represents the fourth-order tensor $B_{i j k l}$, through the relations $I=i \delta_{i j}+(9-i-j), J=k \delta_{k l}+$ $(9-k-l) ; i, j, k, l=1,2,3$. Define a vector $\mathbf{N}=\left(n_{x}, n_{y}, n_{z}\right)$, where $n_{j}$ denotes the components of a unit vector normal to wave surface and hence, it represents the direction of phase propagation. Consider two vectors $\mathbf{U}$ and $\mathbf{V}$ to define the elements of a square matrix $\mathbf{Z}(\mathbf{U}, \mathbf{V})$, as follows:

$$
\begin{aligned}
& Z_{11}=(\mathbf{U} \otimes \mathbf{V}) \cdot \mathbf{A}_{\mathbf{1}}, \quad Z_{22}=(\mathbf{U} \otimes \mathbf{V}) \cdot \mathbf{A}_{\mathbf{2}}, \\
& Z_{33}=(\mathbf{U} \otimes \mathbf{V}) \cdot \mathbf{A}_{\mathbf{3}}, \\
& Z_{12}=(\mathbf{U} \otimes \mathbf{V}) \cdot \mathbf{A}_{\mathbf{4}}, \quad Z_{13}=(\mathbf{U} \otimes \mathbf{V}) \cdot \mathbf{A}_{\mathbf{5}}, \\
& Z_{21}=(\mathbf{U} \otimes \mathbf{V}) \cdot \mathbf{A}_{\mathbf{6}}, \\
& Z_{23}=(\mathbf{U} \otimes \mathbf{V}) \cdot \mathbf{A}_{\mathbf{7}}, \quad Z_{31}=(\mathbf{U} \otimes \mathbf{V}) \cdot \mathbf{A}_{\mathbf{8}}, \\
& Z_{32}=(\mathbf{U} \otimes \mathbf{V}) \cdot \mathbf{A}_{\mathbf{9}},
\end{aligned}
$$

where $\mathbf{A}_{\mathbf{j}},(j=1,2, \ldots, 9)$, the second order tensors are given by

$$
\begin{aligned}
& \mathbf{A}_{\mathbf{1}}=\left\{b_{11}, b_{16}, b_{15} ; b_{61}, b_{66}, b_{65} ; b_{51}, b_{56}, b_{55}\right\} ; \\
& \mathbf{A}_{\mathbf{2}}=\left\{b_{66}, b_{62}, b_{64} ; b_{26}, b_{22}, b_{24} ; b_{46}, b_{42}, b_{44}\right\} ; \\
& \mathbf{A}_{\mathbf{3}}=\left\{b_{55}, b_{54}, b_{53} ; b_{45}, b_{44}, b_{43} ; b_{35}, b_{34}, b_{33}\right\} ; \\
& \mathbf{A}_{\mathbf{4}}=\left\{b_{16}, b_{12}, b_{14} ; b_{66}, b_{62}, b_{64} ; b_{56}, b_{52}, b_{54}\right\} ; \\
& \mathbf{A}_{\mathbf{5}}=\left\{b_{15}, b_{14}, b_{13} ; b_{65}, b_{64}, b_{63} ; b_{55}, b_{54}, b_{53}\right\} ; \\
& \mathbf{A}_{\mathbf{6}}=\left\{b_{61}, b_{66}, b_{65} ; b_{21}, b_{26}, b_{25} ; b_{41}, b_{46}, b_{45}\right\} ; \\
& \mathbf{A}_{\mathbf{7}}=\left\{b_{65}, b_{64}, b_{63} ; b_{25}, b_{24}, b_{23} ; b_{45}, b_{44}, b_{43}\right\} ; \\
& \mathbf{A}_{\mathbf{8}}=\left\{b_{51}, b_{56}, b_{55} ; b_{41}, b_{46}, b_{45} ; b_{31}, b_{36}, b_{35}\right\} ; \\
& \mathbf{A}_{\mathbf{9}}=\left\{b_{56}, b_{52}, b_{54} ; b_{46}, b_{42}, b_{44} ; b_{36}, b_{32}, b_{34}\right\} .
\end{aligned}
$$

Non-trivial solution of Christoffel equation (5) requires to satisfy an equation, given by

$$
\operatorname{det}(\mathbf{X}-h \mathbf{I})=0
$$

where $\mathbf{I}$ is the identity matrix of order three. The square symmetric matrix

$$
\mathbf{X}=\frac{[\mathbf{Z}(\mathbf{N}, \mathbf{N})-\boldsymbol{\Sigma}+\boldsymbol{\Sigma}(\mathbf{N} \otimes \mathbf{N})-(\mathbf{N} \otimes \mathbf{N}) \mathbf{\Sigma}]}{\rho}
$$

where matrix $\boldsymbol{\Sigma}=\frac{1}{2}\left\{S_{i j}\right\}$ and

$$
h=v^{2}-(\mathbf{N} \otimes \mathbf{N}) \cdot \frac{\boldsymbol{\Sigma}}{\rho} .
$$

The characteristic equation (17) is further solved into a cubic equation in $h$, given by

$$
h^{3}+a h^{2}+b h+c=0
$$

where,

$$
\begin{aligned}
a= & -\left(X_{11}+X_{22}+X_{33}\right) ; \\
b= & X_{11} X_{22}+X_{11} X_{33}+X_{22} X_{33} \\
& -X_{12}^{2}-X_{13}^{2}-X_{23}^{2} ; \\
c= & X_{11} X_{23}^{2}+X_{22} X_{13}^{2}+X_{33} X_{12}^{2} \\
& -X_{11} X_{22} X_{33}-2 X_{12} X_{13} X_{23} .
\end{aligned}
$$

The three roots of this polynomial equation explain the propagation of three quasi-waves in a prestressed anisotropic elastic medium. The roots of this equation are given by

$$
\begin{aligned}
& h_{1}=L+M-\frac{a}{3}, \quad h_{2}=L \Omega^{2}+M \Omega-\frac{a}{3}, \\
& h_{3}=L \Omega+M \Omega^{2}-\frac{a}{3},
\end{aligned}
$$

where $\Omega=(-1+\imath \sqrt{3}) / 2, L=(-G+\Delta)^{1 / 3}$, and $M=(-G-\Delta)^{1 / 3}, \quad$ and $\quad \Delta=\sqrt{\left.G^{2}-H^{3}\right)}$ for $H=\left(a^{2}-3 b\right) / 9$ and $G=\left(2 a^{3}-9 a b+27 c\right) / 54$. Corresponding to three roots of (20), $v_{m}=$ $\sqrt{h_{m}+(\mathbf{N} \otimes \mathbf{N}) \cdot \boldsymbol{\Sigma} / \rho},(m=1,2,3)$, define the phase velocities of three quasi-waves propagating in the direction of vector $\mathbf{N}$. These waves, represented by $m=1,2$ and 3 , are called the $q P, q S 1$ and $q S 2$ waves respectively.

In an anisotropic medium, energy associated with a wave travel with the group velocity along a ray at an angle to its direction of phase propagation. In a spherical co-ordinate system, $v(\theta, \phi)$ 
define the phase velocity of a wave in the vertical plane ( $\phi=$ constant) along the phase direction which is making an angle $\theta$ with the polar axis. The component of group velocity normal to interface $x_{3}=0$, is then expressed as

$$
g_{3}=v\left(\cos \theta-\sin \theta T_{\theta}\right),
$$

where,

$$
T_{\theta}=\frac{1}{v}(v)_{, \theta}=\frac{1}{2 v^{2}}\left(\frac{h_{, \theta}+2\left(\mathbf{N}_{, \theta} \otimes \mathbf{N}\right) \cdot \boldsymbol{\Sigma}}{\rho}\right) .
$$

The partial derivatives of $h$ are derived, analytically from cubic equation (20).

\section{Appendix B: Christoffel equation in slowness}

Two vectors $\mathbf{U}$ and $\mathbf{V}$ define a square matrix $\mathbf{Y}(\mathbf{U}, \mathbf{V})$, as follows.

$$
\begin{aligned}
\mathbf{Y}(\mathbf{U}, \mathbf{V})= & \mathbf{Z}(\mathbf{U}, \mathbf{V})+\boldsymbol{\Sigma}(\mathbf{U} \otimes \mathbf{V})-(\mathbf{U} \otimes \mathbf{V}) \boldsymbol{\Sigma} \\
& +[(\mathbf{U} \otimes \mathbf{V}) \cdot \boldsymbol{\Sigma}] \mathbf{I}-\boldsymbol{\Sigma}(\mathbf{U} \cdot \mathbf{V}), \quad(25)
\end{aligned}
$$

where $\mathbf{Z}(\mathbf{U}, \mathbf{V})$ and $\boldsymbol{\Sigma}$ are same as defined in Appendix A. Consider two vectors $\mathbf{P}=\left(p_{1}, p_{2}, 0\right)$ and $\mathbf{Q}=(0,0,1)$ to define three square matrices

$$
\begin{aligned}
& \mathbf{A}=\mathbf{Y}(\mathbf{P}, \mathbf{P}), \\
& \mathbf{B}=\mathbf{Y}(\mathbf{P}, \mathbf{Q})+\mathbf{Y}(\mathbf{Q}, \mathbf{P}), \\
& \mathbf{C}=\mathbf{Y}(\mathbf{Q}, \mathbf{Q})-\rho \mathbf{I} .
\end{aligned}
$$

Then, the coefficients $\left(\alpha_{j}\right)$ in equation (7) are expressed as follows:

$$
\begin{aligned}
\alpha_{6}= & |A A A| ; \quad \alpha_{5}=|A A B|+|A B A|+|B A A| ; \\
\alpha_{4}= & |A A C|+|A C A|+|C A A|+|A B B| \\
& +|B A B|+|B B A| ; \\
\alpha_{3}= & |A B C|+|A C B|+|B A C|+|B C A| \\
& +|C A B|+|C B A|+|B B B| ; \\
\alpha_{2}= & |A C C|+|C A C|+|C C A|+|B B C| \\
& +|B C B|+|C B B| ; \\
\alpha_{1}= & |B C C|+|C B C|+|C C B| ; \\
\alpha_{0}= & |C C C|,
\end{aligned}
$$

where the symbol $|A B C|$ denotes the determinant of a matrix obtained by selecting first row from matrix $\mathbf{A}$, second row from matrix $\mathbf{B}$ and third row from matrix $\mathbf{C}$.

\section{References}

Biot M A 1939 Non-linear theory of elasticity and the linearized case for a body under initial stress; Phil. Mag. $\mathbf{2 7}$ 468-489.

Biot M A 1940 Influence of initial stress on elastic waves; J. Appl. Phys. 11 522-530.

Biot M A 1965 Mechanics of Incremental Deformation; John Wiley, New York.

Borcherdt R D 1977 Reflection and refraction of type-II $\mathrm{S}$ waves in elastic and inelastic media; Bull. Seismol. Soc. Am. 67 43-67.

Chai J-F and Wu T-T 1996 Determination of surface wave velocities in a prestressed anisotropic solid; NDT $\mathscr{E} E$ Int 29 281-292.

Crampin S 1981 A review of wave motion in anisotropic and cracked elastic media; Wave Motion 3 343-391.

Degtyar A D and Rokhlin S I 1995 Absolute stress determination in orthotropic materials from angular dependence of ultrasonic velocities; J. Appl. Phys. $\mathbf{7 8}$ 1547-1556.

Degtyar A D and Rokhlin S I 1997 Stress effect on ultrasonic wave propagation through the solid-solid and liquid-solid plane interface; In: Reviews of Progress in Quantitative NDE (eds) D O Thompson and D E Chimenti, Plenum, New York 16B 1699-1706.

Degtyar A D and Rokhlin S I 1998 Stress effect on boundary conditions and elastic wave propagation through an interface between anisotropic media; J. Acoust. Soc. Am. 104 1992-2003.

Gibson Jr R L and Toksoz M N 1990 Permeability estimation from velocity anisotropy in fractured rocks; J. Geophys. Res. 95 15,643-15,656.

Hanks B C and Raleigh C B 1980 The conference on magnitude of deviatoric stresses in the earth's crust and uppermost mantle; J. Geophys. Res. 85 $6083-6085$.

Keith C M and Crampin S 1977 Seismic body waves in anisotropic media: reflection and refraction at a plane interface; Geophys. J. R. Astr. Soc. 49 181-208.

Lanceleur P, Ribeiro H and De Belleval J-F 1993 The use of inhomogeneous waves in the reflection-transmission problem at a plane interface between two anisotropic media; J. Acoust. Soc. Am. 93 1882-1892.

Man C-S and Lu W Y 1987 Towards an acoustoelastic theory for measurements of residual stress; J. Elast. 17 159-182.

McGarr A 1980 Some constraints on levels of shear stress in the crust from observation and theory; J. Geophys. Res. 85 6231-6238.

Norris A N and Sinha B K 1995 Speed of a wave along a fluid/solid interface in the presence of anisotropy and prestress; J. Acoust. Soc. Am. 98 1147-1154.

Prikazchikov D A and Rogerson G A 2003 Some comments on the dynamic properties of anisotropic and strongly anisotropic pre-stressed elastic solids; Int. J. Engg. Sci. 41 149-171.

Rasolofosaon P N J and Zinszner B E 2002 Comparison between permeability anisotropy and elasticity anisotropy of reservoir rocks; Geophysics 67 230-240.

Rokhlin S I, Boland T K and Adler L 1986 Reflection and refraction of elastic waves on a plane interface between 
two generally anisotropic media; J. Acoust. Soc. Am. 79 906-918.

Sayers C M, Johnson G M and Denyer G 2002 Predrill pore-pressure prediction using seismic data; Geophysics 67 1286-1292.

Sharma M D 2005 Effect of initial stress on the propagation of plane waves in a general anisotropic poroelastic medium; J. Geophys. Res. 110 B11307.

Sharma M D and Garg N 2006 Wave velocities in a prestressed anisotropic elastic medium; J. Earth Syst. Sci. $115257-265$.
Sharma M D and Gogna M L 1991 Propagation of Love waves in an initially stressed medium consisting of slow elastic layer lying over a liquid-saturated porous solid half-space; J. Acoust. Soc. Am. $\mathbf{8 9}$ $2584-2588$.

Spencer A J M 1941 Deformation of fibre-reinforced materials, Clarendon Press, Oxford.

Synge J L 1957 Elastic waves in anisotropic media; J. Math. Phys. 35 323-334.

Tolstoy I 1982 On elastic waves in prestressed solid; J. Geophys. Res. 87 6823-6827. 